Ann. Biol. anim. Bioch. Biophys., 1977, 17 (6), 1047-1060.

\title{
Technique de réalisation d'une poche abomasale chez le veau en vue de l'obtention de suc gastrique pur
}

\author{
par P. GUILLOTEAU, J. L. LE CALVE * \\ avec la collaboration technique de S. BOUSSION, Monique LESNE, Y. MANIS \\ Station de Recherches Zootechniques, I.N.R.A., \\ E. N. S. A., 65, rue de St-Brieuc, 35042 Rennes Cedex. \\ * Clinique Chirurgicale B, Unité de Chirurgie digestive \\ et Transplaniation C. H.U. 35000 Rennes
}

Summary. A technique for the preparation of calf abomasal pouches to obtain pure gastric juice.

We have described a technique for preparing an innervated abomasal pouch with a view to obtaining pure calf gastric juice. The gastric pouch was cut out from the middle part of the lesser abomasal curvature to a depth of approximately $10 \mathrm{~cm}$, leaving the blood vessels and nerves intact. It was placed in a zone with numerous folds of mucous membrane spreading out in the fundus (fig. 1, 2, 3, 4, 5 and 6).

Some aspects of the anatomy of the abomasum, as well as details on the anesthesia, type of catheter used, post-operative care and difficulties encountered in the preparation of abomasal pouches are also discussed.

The first three operated calves were used later in good conditions for 34, 85 and 228 days respectively (table 1), while still maintaining a satisfactory growth pattern and sanitary state. The daily amounts of gastric juice secreted by the pouch varied from 446 to $1552 \mathrm{~g} /$ $100 \mathrm{~kg}$ of live weight (fig. 7).

\section{Introduction.}

Chez le monogastrique, les sécrétions gastriques peuvent être prélevées in vivo par incubation de l'estomac. Cette technique est plus difficile à mettre en œuvre chez le Veau puisque c'est un polygastrique. Aussi, chez cet animal, la fistulisation de la caillette avec pose d'une canule, est-elle la méthode la plus employée (Berridge ef al., 1943 ; Nair, Mehar Singh ef Bhalerao, 1963 ; Cisneros ef al., 1972 ; Garnot ef al., 1977). De réalisation rapide, elle est la plus simple des préparations gastriques innervées. Ces deux techniques présentent des inconvénients qui en limitent l'utilisation : elles n'évitent pas la contamination salivaire directe et bilio-pancréatique rétrograde 
du suc gastrique, elles ne permettent pas une étude cinétique de la sécrétion, et surtout la fuite du contenu gastrique par le pylore est possible (Mignon et Bonfils, 1972).

Il est souhaitable d'utiliser une autre technique qui permette de recueillir du suc gastrique pur en restant aussi près que possible des conditions physiologiques, chez l'animal non anesthésié ; c'est ainsi que la préparation d'une poche abomasale semble satisfaire en partie ces objectifs. Des poches gastriques de nombreux types sont réalisées chez le Chien (Emas, Swan et Jacobson, 1967 ; Konturek, 1974). Elles sont découpées soit sur la grande courbure de l'estomac (poches de Pavlov ou de Heidenhain), soit dans la région de l'antre pylorique (poches pylorique ou de Forrest), soit encore en utilisant l'estomac dans sa totalité après anastomose œesophago-duodénale (poches de Ivy, Lim et McCarty, 1925). A notre connaissance, seul Amour (1930) décrit une poche gastrique découpée sur la petite courbure. Chez le Veau ef le Mouton, quelques études seulement sont faites en utilisant des animaux porteurs d'une poche d'Heidenhain ou de Pavlov (Ash, 1961 ; McLeay et Titchen, 1970 ; Rusev, Petkov ef Bocheva, 1971). Grosskopf (1954) propose une opération simplifiée pour préparer une poche sur la grande courbure de la caillette du Veau. Hill et Gregory (1951) décrivent différents types de poches abomasales chez la Chèvre.

Dans ce travail, nous proposons un type de poche abomasale (ou petit estomac), découpée sur la petite courbure de la caillette du Veau, et différente de celle d'Amour (1930).

\section{Matériel et méthodes.}

Animaux. Des veaux mâles de race Frisonne sont utilisés. Achetés à l'âge d'environ 8 jours, ils reçoivent un lait de remplacement à raison de 2 repas par jour, et sont alimentés en fonction de leur poids métabolique. La totalité des protéines de l'aliment d'allaitement est apportée par du lait écrémé. Les veaux sont ainsi maintenus au stade préruminant. Après une période d'observation d'une semaine au minimum, chaque animal est préparé pour l'intervention. Il est mis à jeûn la veille au soir et le jour de l'opération. Après celle-ci, il esł progressivement réalimenté pour atteindre son niveau d'ingestion antérieur.

Rappels d'anatomie. La caillette (ou abomasum) du veau correspond à l'estomac du monogastrique avec lequel elle présente une grande analogie analomique. La vascularisation de l'estomac dérive du tronc cœliaque. Les artères gastriques droite et gauche forment le cercle artériel de la petite courbure de la caillette, tandis que les artères gastroépiploïques droite et gauche constituent le cercle artériel de la grande courbure (fig. 1). La distribution intramurale est assurée par un riche réseau sousmuqueux. Les veines sont satellites des artères et sont draînées par le système porte (Comline, Silver et Steven, 1968 ; Habel, 1975 ; Schummer ef al, 1976).

L'innervation parasympathique est assurée par les branches des deux nerfs pneumogastriques : une branche du tronc vagal dorsal innerve la face dorsale de la caillette, une branche du tronc ventral la face ventrale. Ces rameaux cheminent de part ef d'autre de la petite courbure, dans le petit épiploon (fig. 1). L'innervation sympathique de la caillette est principalement réalisée par des fibres en provenance du plexus coeliaque (Comline, Silver et Steven, 1968 ; Habel, 1975). 


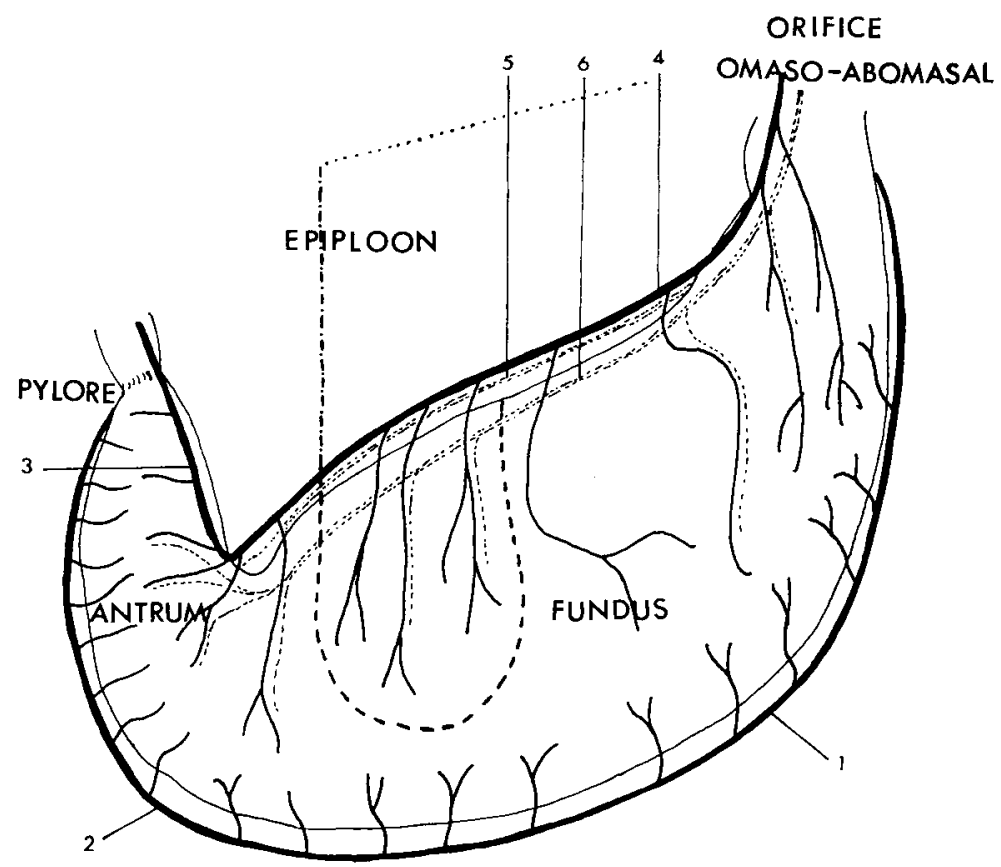

FIG. 1. - Anatomie de la caillette (face ventrale). Emplacement de la poche abomasale.

1. - Artère gastro-épiploïque gauche.

2. - Artère gastro-épiploĩque droite.

3. - Artère gastrique droite.

4. - Artère gastrique gauche.

5. - Branche du tronc vagal dorsal.

6. - Branche du trone vagal ventral.

..... Incision dans le petit épiploon à distance de la petite courbure.

_._. - Section entre ligatures des éléments vasculo-nerveux et section complète du petit épiploon qui permettra la mobilisation de la poche.

- - Emplacement de la poche abomasale.

\section{Technique opératoire.}

- Un cathéter est préparé à partir d'un tube en silastic $R\left({ }^{1}\right)$, dont les diamètres intérieur et extérieur sont respectivement de 6,4 et $9,5 \mathrm{~mm}$ ef la longueur d'environ $50 \mathrm{~cm}$. Ce tube est muni à l'une de ses extrémités, d'une bague de $2 \mathrm{~cm}$ de diamètre faite en élastomère de silicone $\left({ }^{2}\right)$. Cette bague sera placée à l'intérieur de la poche abomasale.

- L'anesthésie générale, avec infubation trachéale, est induite par l'injection intraveineuse lente de pentothal $(10 \mathrm{mg} / \mathrm{kg}$ de poids vif) et entretenue en utilisant de

(1) Réf. 601-445 : Dow Corning, 36-38, rue de la Princesse, 78430 Louveciennes.

(2) Réf. 890 : Dow Corning, 36-38, rue de la Princesse, 78430 Louveciennes. 

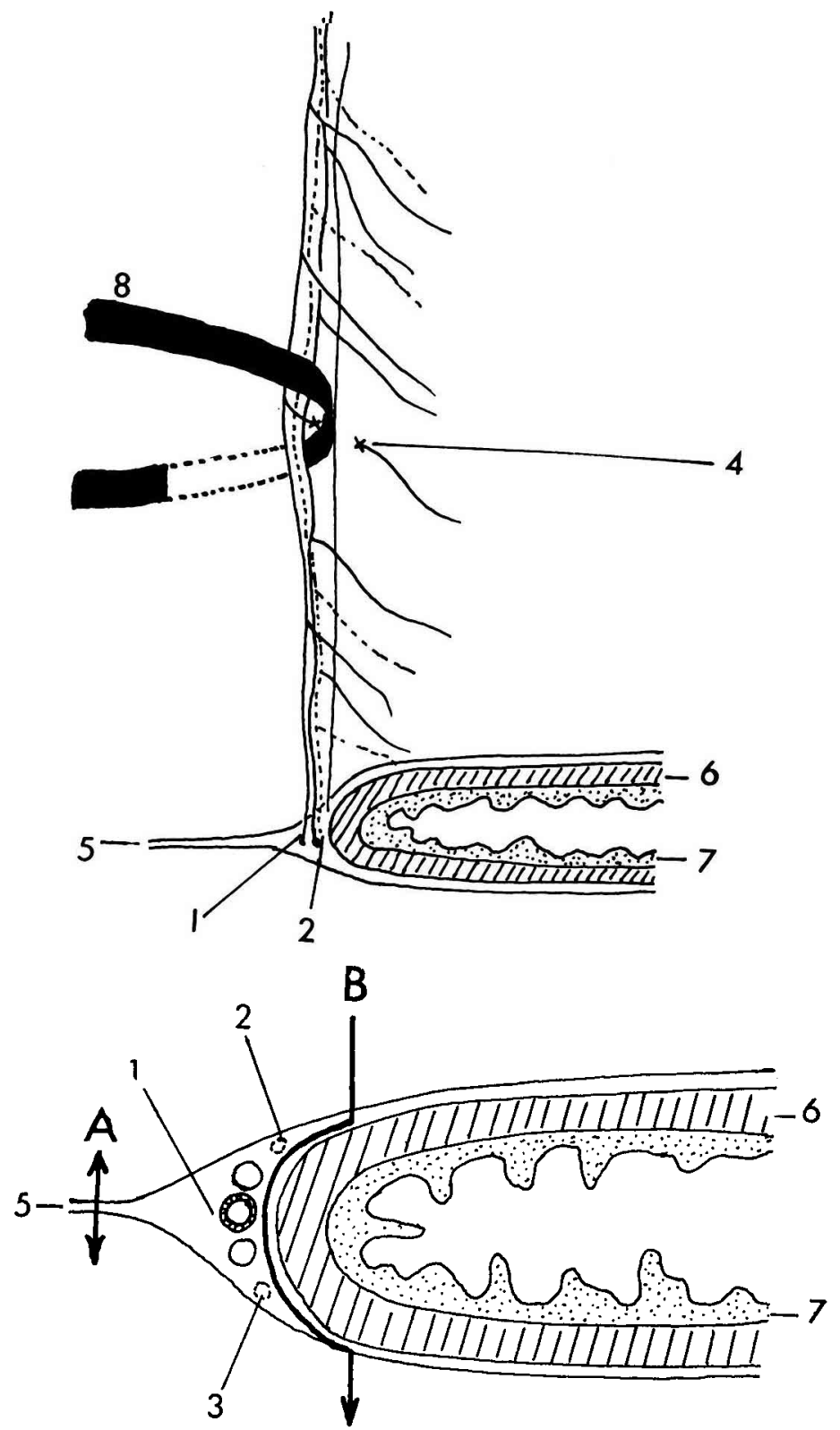

FIG. 2. - Individualisation du pédicule vasculo-nerveux du futur petit estomac.

1. - Pédicule vasculaire gauche.

2. - Branche du tronc vagal ventral.

3. - Branche du tronc vagal dorsal.

4. - Vaisseau droit suturé.
5. - Petit épiploon.

6. - Séro-musculeuse de la caillette.

7. - Muqueuse de la caillette.

8. - Compresse stérile.

A : Incision dans le petit épiploon à distance de la petite courbure.

B : incision péritonéale pratiquée sur les deux faces à proximité. La dissection doit se faire en rasant le bord de la caillette. 
l'halothane en circuit fermé ; l'assistance respiratoire est assurée à l'aide d'un appareil Bird-Mark 4.

- L'opération chirurgicale proprement dite se décompose en plusieurs temps opératoires :

L'ouverture de la paroi abdominale se fait par un abord médian sus-ombilical (le long de la ligne blanche) débordant éventuellement l'ombilic à droite (section entre ligatures du ligament rond). Un écarteur autostatique est mis en place, après avoir assuré une protection pariétale par deux champs de bordure. La caillette est alors exposée, son extériorisation étant aisée.

Les éléments vasculo-nerveux (pédicule vasculaire gastrique gauche, branches du tronc vagal dorsal et du trone vagal ventral avec leurs ramifications) sont repérés dans le petit épiploon après incision de celui-ci à distance de la petite courbure (fig. 1). Il en résulte une détermination précise de l'emplacement de la poche abomasale. Celle-ci est découpée sur les deux faces de la caillette à partir de la petite courbure en sa partie moyenne, sur une hauteur d'environ $10 \mathrm{~cm}$, en respectant la vascularisation et l'innervation (fig. 1). Elle se situe dans une zone où la muqueuse présente de nombreux replis et intéresse surtout le fundus.

Au bord oral de la poche, il est nécessaire d'individualiser le pédicule vasculonerveux du futur petit estomac, en respectant les éléments vasculaires gastriques gauches et les branches des troncs dorsal et ventral des nerfs pneumogastriques. Pour cela, une incision péritonéale est pratiquée sur les deux faces à proximité de la petite courbure, après ligature éventuelle d'un ou plusieurs vaisseaux droits. La dissection doit se faire en rasant le bord de la caillette (fig. 2). Au bord aboral de la poche, un geste semblable est réalisé ; de plus, on pratique une section entre ligatures des éléments vasculo-nerveux, ainsi qu'une section complète de l'épiploon qui permettra la mobilisation de la poche.

Après la mise en place de quatre clamps souples respectant le pédicule vasculonerveux (fig. 3) une section entre clamps extérieur et intérieur est réalisée (fig. 4). Quelques hémostases complémentaires sur la tranche de section peuvent être nécessaires et sont faites à l'aiguille sertie. La poche abomasale, délimitée par les deux clamps souples intérieurs, est basculée autour du pédicule vasculo-nerveux la desservant. Ainsi, l'opérateur a libre accès aux tranches de section de la caillette proprement dite (fig. 4). Pour reconstituer la caillette, les deux clamps souples extérieurs sont rapprochés l'un de l'autre avec beaucoup de précautions de façon à mettre en contact les deux tranches (fig. 5). La technique de suture bord à bord de chacune des deux faces se fait en deux plans avec du fil non résorbable serti (déc. 3) : un plan muqueux par un surjet simple à visée hémostatique et un plan séro-musculaire extramuqueux à points simples ou en surjet (fig. 5). Il est important d'avoir une parfaite exposition des deux berges à suturer, ce qui implique que les clamps soient suffisamment écartés avant la découpe de la poche ( $2 \mathrm{~cm}$ environ).

La poche est suturée selon le même principe, le cathéter étant extériorisé au sommet de celle-ci. On termine par une bourse d'enfouissement au point de sortie du cathéter, assurant l'étanchéité et évitant le rejet de ce dernier (fig. 6).

Après avoir réintégré la caillette, le cathéter est extériorisé du côté droit, là où la poche abomasale vient le plus naturellement au contact de la paroi abdominale et 

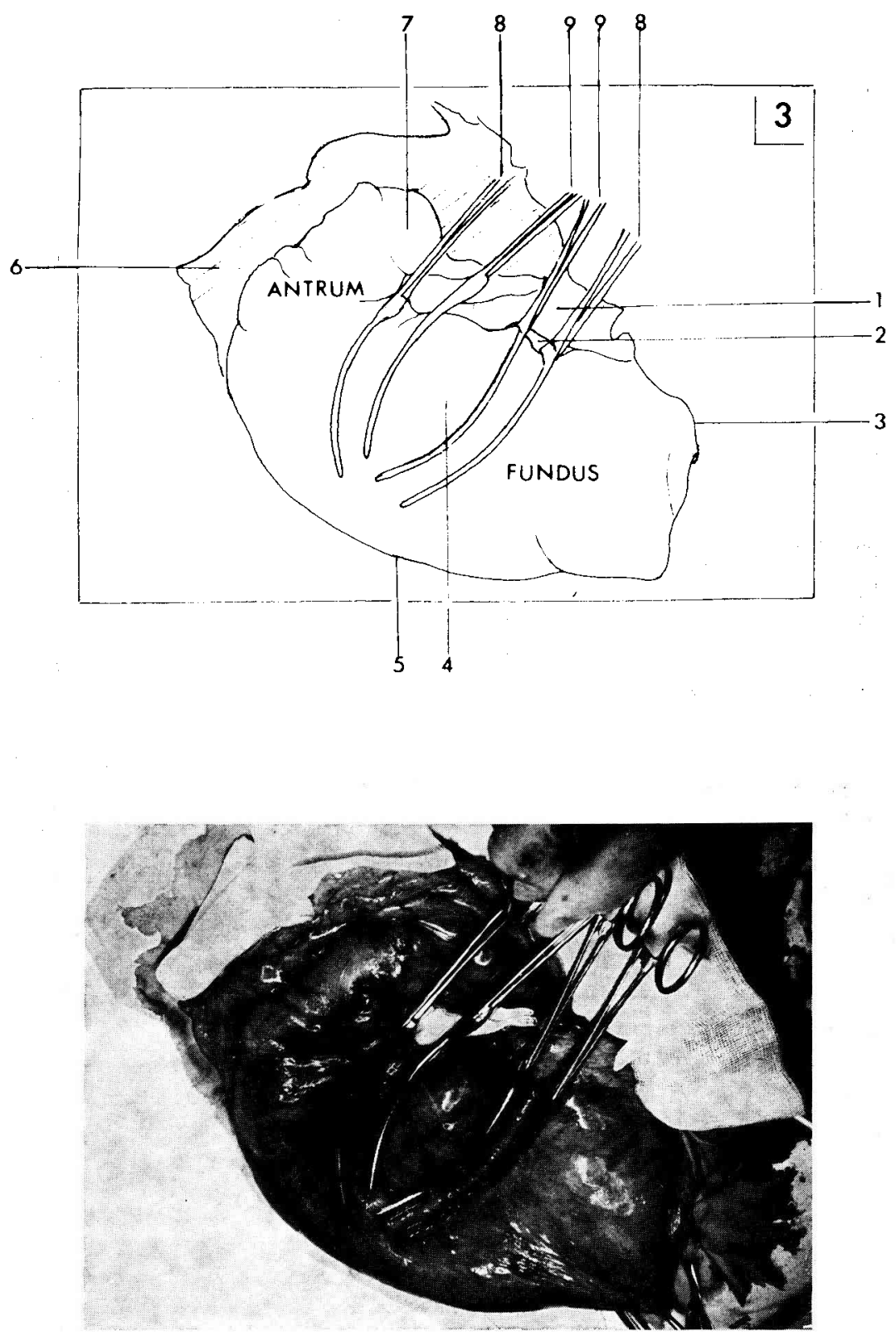

PLANCHE I

FIG. 3. - Mise en place des quatre clamps souples en respectant le pédicule vasculo-nerveux.

FIG. 4. - Après la section entre clamps, la poche abomasale est basculée autour du pédicule contenant le tronc vasculo-nerveux qui la dessert. 

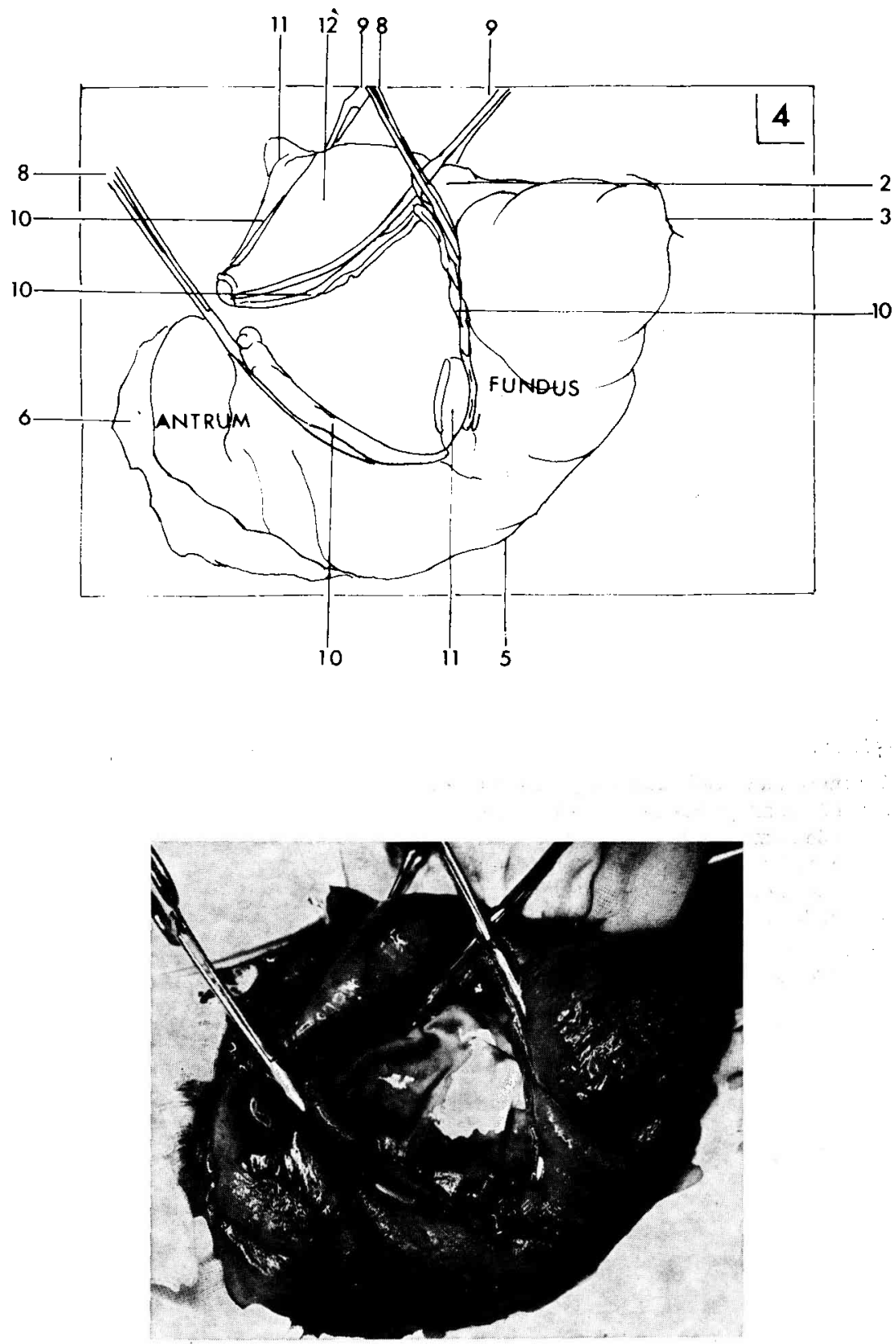

1. - Petit épiploon sectionné et suturé. 2. - Pédicule vasculo-nerveux. 3. - Paroi abdominale coupée. 4. - Future poche abomasale. 5. - Grande courbure de la caillette. 6. - Grand épiploon. 7. - Pylore. 8. - Clamps souples extérieurs. 9. - Clamps souples intérieurs. 10. - Tranches de section. 11. - Feuillets muqueux. 12. - Poche abomasale découpée. 


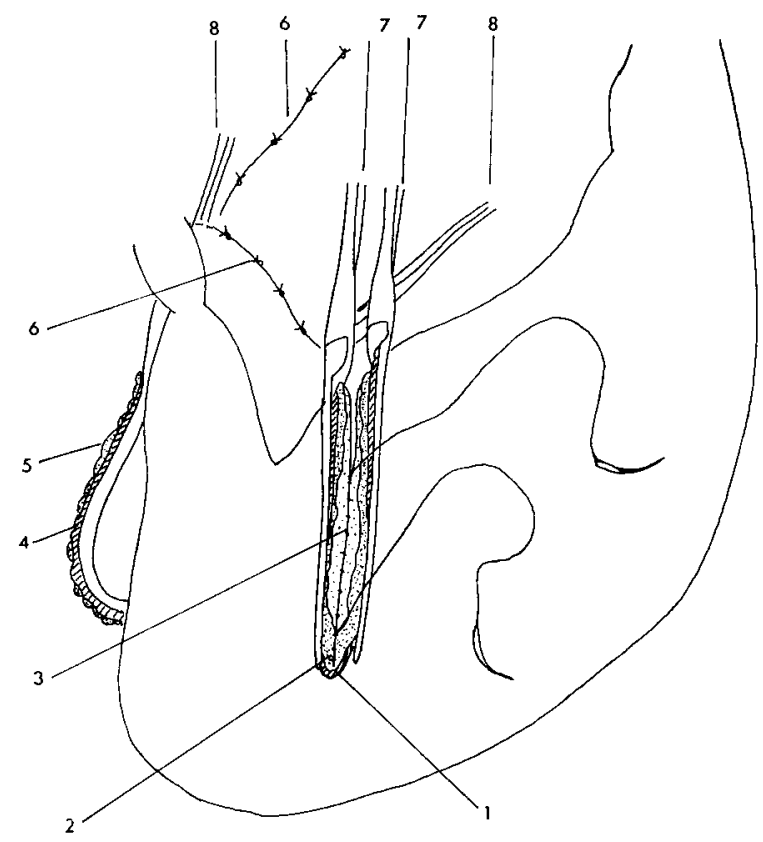

FIG. 5. - Reconstitufion de la caillette proprement dife : suture bord à bord du plan muqueux (surjet simple à visée hémosfatique) après avoir rapproché l'un de l'autre les deux clamps souples extérieurs.

1. - Séro-musculeuse de la caillette (face ventrale).

2. - Suture de la muqueuse de la face ventrale.

3. - Suture de la muqueuse de la face dorsale.

4. - Séro-musculeuse de la poche abomasale (face ventrale).

5. - Muqueuse de la poche abomasale.

6. - Petit épiploon sectionné et suturé.

7. - Clamps souples extérieurs.

8. - Clamps souples intérieurs.

en position déclive. L'extrémité du petit estomac est ramenée contre la paroi abdominale, dans le but d'éviter qu'une anse intestinale ne vienne s'étrangler autour du tube de silastic. De plus, il est nécessaire de faire très attention à ne pas provoquer une torsion du pédicule vasculo-nerveux, car ceci comporte un risque de nécrose par ischémie.

La paroi abdominale est refermée en suturant plan par plan avec des fils de nylon $n^{0} 6$ (ensemble péritoine-aponévrose) et no 8 (p€au). A l'extérieur de l'abdomen, le cathéter est attaché solidement à la peau par un fil de nylon no 8 . Une poche stérile de récolte à urine $\left(^{3}\right)$ est fixée à l'extrémité du cathéter et permet de recueillir les sécrétions du petit estomac ; elle est changée périodiquement. L'ensemble cathéter et poche de collecte est plaqué contre la paroi abdominale à l'aide d'un filet élastique $\left(^{4}\right)$ entourant complètement l'animal au niveau de l'abdomen.

$\left({ }^{3}\right)$ Poly-Labo P. Block ef Cie, 303a, Route de Colmar, BP 201, 67022 Strasbourg.

(4) Calibre $6:$ U. V. A., 20, rue Lebrun, 75013 Paris. 
Les soins per et post-opératoires ont pour but de prévenir les infections péritonéales. Au moment de l'opération, la cavité abdominale est saupoudrée à l'aide de 1 million d'Ul de pénicilline avant la suture. Après l'opération, quatre injections intramusculaires contenant du chloramphénicol $(0,8 \mathrm{~g})$ ef du chlorhydrate de tétracycline $(0,3 \mathrm{~g})$ sont administrées à $24 \mathrm{~h}$ d'intervalle. Durant toute l'expérience, l'animal est surveillé de façon attentive (température rectale, changement fréquent des pansements, observations des plaies, etc...) afin de déceler précocement une infection éventuelle.

\section{Résultats et discussion.}

La technique chirurgicale décrite permet d'isoler de la caillette un petit estomac qui, au moment de l'opération représente $1 / 4$ à $1 / 8$ de sa surface totale. Le reste de la cailletfe est encore relativement important et doit pouvoir assurer une digestion gastrique normale.

L'état sanitaire des 3 premiers animaux opérés a été satisfaisant et leur croissance a été voisine de celle observée chez les veaux préruminants (tabl. 1). Le cathéter est resté fonctionnel pendant au moins 82 jours ; des fuites se sont établies progressivement autour du cathéter du deuxième ef du troisième animal, respectivement au cours de la trente troisième et de la treizième semaine après l'opération. Grosskopf (1959) observe le même phénomène chez des veaux, 3 à 5 semaines après la mise en place de la poche abomasale.

TABLEAU 1

Performances des 3 premiers animaux munis d'une poche abomasale, selon la technique proposée

\begin{tabular}{|c|c|c|c|c|c|}
\hline \multirow[b]{2}{*}{ Veau $n^{\circ}$} & \multirow{2}{*}{$\begin{array}{l}\text { Age } \\
\text { à l'opération } \\
\text { (i) }\end{array}$} & \multirow{2}{*}{$\begin{array}{c}\text { Age } \\
\text { à l'abattage } \\
\text { (j) }\end{array}$} & \multicolumn{2}{|c|}{ Période expérimentale } & \multirow{2}{*}{$\begin{array}{l}\text { Causes de l'arrêt } \\
\text { de la période } \\
\text { expérimentale }\end{array}$} \\
\hline & & & $\begin{array}{l}\text { Durée } \\
\text { (i) }\end{array}$ & $\begin{array}{l}\text { Gain de } \\
\text { poids vif } \\
(\mathrm{g} / \mathrm{j})\end{array}$ & \\
\hline 76558 & 46 & 128 & 34 & 882 & $\begin{array}{l}\text { Intoxication alimentaire à la suite } \\
\text { d'un changement de régime }\end{array}$ \\
\hline $\begin{array}{l}76625 \\
76635\end{array}$ & $\begin{array}{l}16 \\
28\end{array}$ & $\begin{array}{l}254 \\
146\end{array}$ & $\begin{array}{r}228 \\
85\end{array}$ & $\begin{array}{l}1032 \\
1026\end{array}$ & $\begin{array}{l}\text { Abattage à la suite d'une fuite } \\
\text { de suc gastrique au niveau de } \\
\text { la fistule. }\end{array}$ \\
\hline
\end{tabular}

La sécrétion apparaît dans le cathéter dès sa pose. Au début, elle est sanglante puis s'éclaircit rapidement et une semaine environ après l'opération, elle est incolore et limpide, avec quelques particules en suspension. Les quantités sécrétées sont relativement importantes et varient selon l'animal et son âge de 100 à plus de $2400 \mathrm{~g} / \mathrm{J}$ 


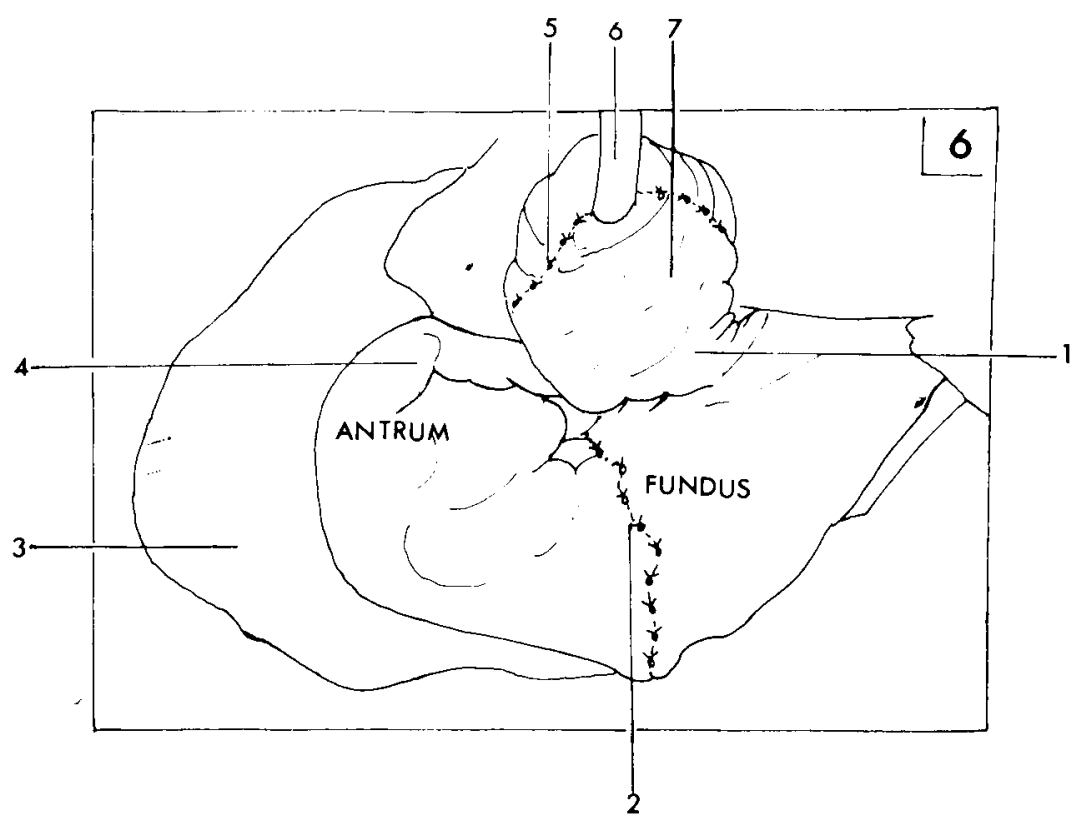

PLANCHE 2

FIG. 6. - La caillefte proprement dite et la poche abomasale sont sufurées.

1. - Pédicule vasculo-nerveux.

2. - Ligne de suture de la caillette proprement dite.

3. - Grand épiploon.

4. - Pylore.

5. - Ligne de suture de la poche abomasale.

6. - Catheter.

7. - Poche abomasale. 


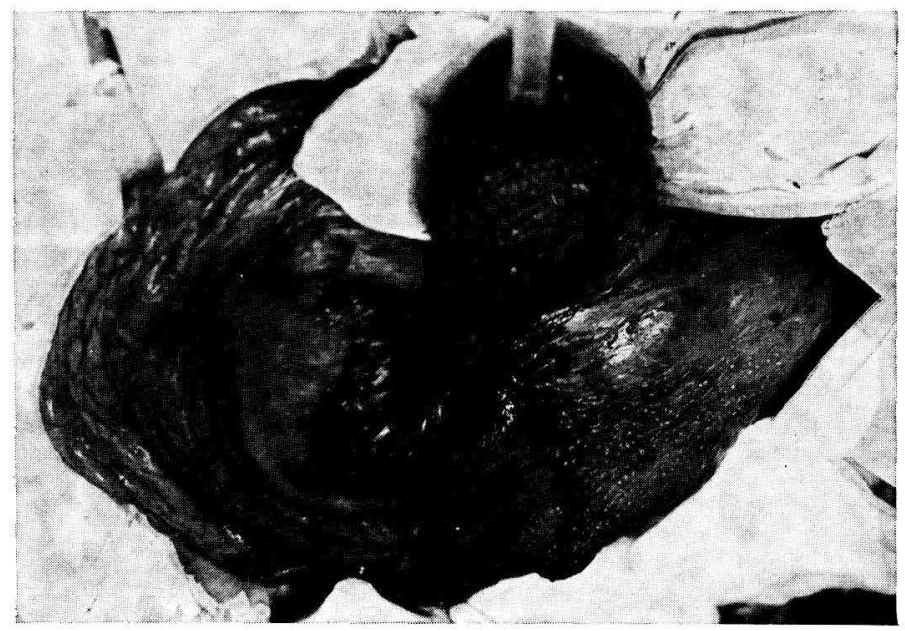

(fig. 7). La quantité totale de suc gastrique sécrétée par le petit estomac semble augmenter progressivement jusqu'à l'âge de 15 à 17 semaines pour rester constante par la suite. Toutefois, les variations journalières sont importantes. Les moyennes hebdomadaires des quantités journalières sécrétées varient de 15 à $47 \mathrm{~g} / \mathrm{kg}$ de poids métabolique (poids vif ${ }^{0,75}$ ) et de 446 à $1552 \mathrm{~g} / 100 \mathrm{~kg}$ de poids vif. Il n'esł pas possible de connaître avec précision la proportion de la sécrétion totale de la caillette qui est effectivement collectée pour chaque veau. Nous pourrions tenter de l'estimer à l'aulopsie en faisant le rapport entre la surface de la muqueuse de la poche et de celle de la caillette. Cependant, du fait de la présence de zones à activités sécrétoires probablement différentes, on peut se demander quelle serait la valeur des comparaisons effectuées ; nous avons donc seulement vérifié le bon état de la muqueuse gastrique. Roy et Stobo (1975) estiment la sécrétion journalière de la caillette du veau préruminant à environ $3 \mathrm{~kg} / 100 \mathrm{~kg}$ de poids vif. Dans nos conditions expérimentales, la fraction recueillie constitue probablement de 15 à 50 p. 100 de la quantité totale sécrétée par la caillette. Il se pourrait que la dérivation de cette part du suc gastrique modifie la sécrétion elle-même ; en effet, Corring (1975) observe que la dérivation de la totalité du suc pancréatique chez le porc a pour effet d'accroître considérablement la quantité sécrétée lorsque le suc n'est pas réintroduit. II sera nécessaire de vérifier l'influence de la réintroduction du suc gastrique recueilli.

La vascularisation ef l'innervation de la poche gastrique sont assurées respectivement par les artère ef veine gastriques gauches ef par les branches du tronc vagal dorsal et du tronc vagal ventral. La variabilité de la fraction du suc gastrique recueilli dépend de l'importance de la poche abomasale elle-même, mais peut être également de son innervation plus ou moins bien respectée. Sans pouvoir répondre avec précision à cette dernière question, nous avons vérifié que les mécanismes réflexes sont maintenus. En effet, lorsqu'on simule un repas (bruit, vue du seau contenant l'aliment sans que l'animal puisse ingérer...), du suc gastrique coule aussitôt par le cathéter. II 


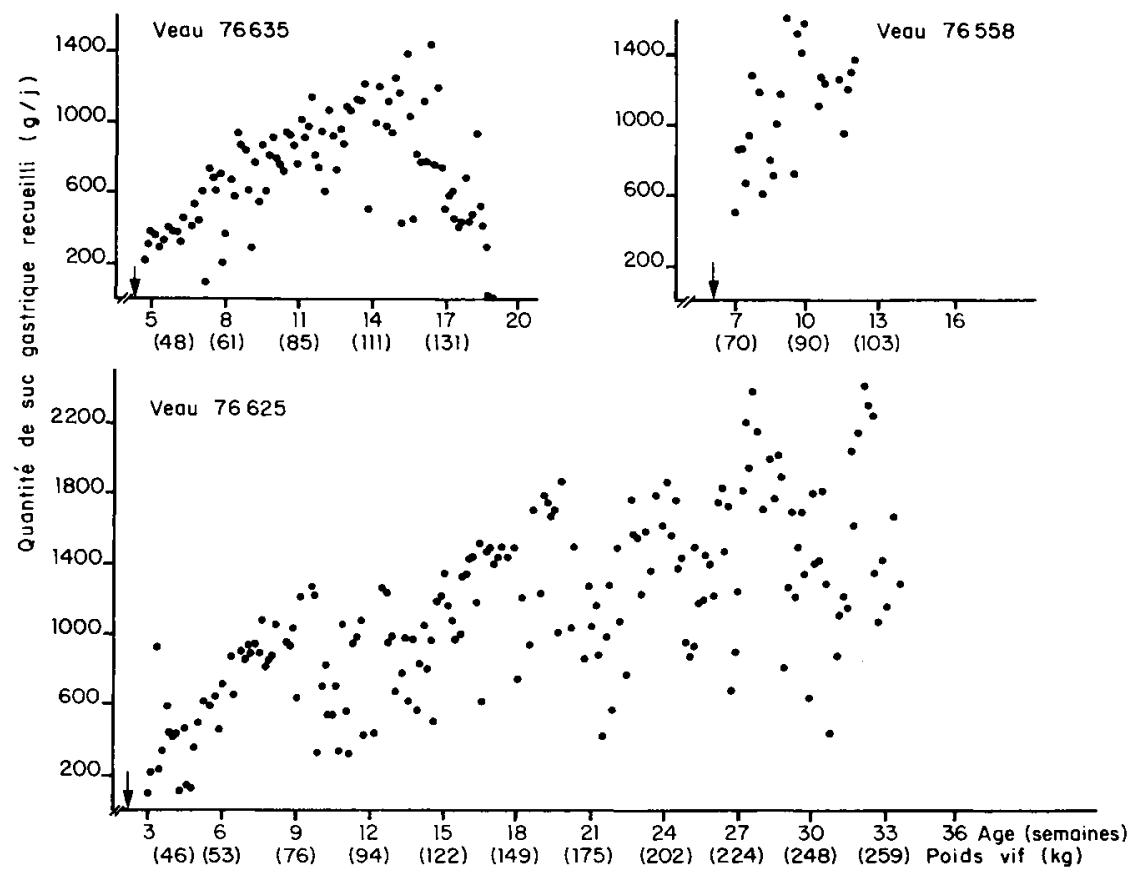

FIG. 7. - Evolution des quantités de suc gastrique recueilli en fonction de l'âge chez les trois animaux en expérience.

$\downarrow$ date de l'opération.

semble que la poche fonctionne comme un petitestomac, dit de Pavlov, fel qu'il est décrit classiquement.

La vascularisation de la caillette conserve son intégrité. La section des filets nerveux parasympathiques au bord aboral de la poche dénerve le territoire plus distal que constitue l'antre et risque de perturber sa fonction (Ruckebusch, communication personnelle). Il est vraisemblable que cette vagotomie partielle modifie la motricité, mais également les réflexes vago-sécrétoires de l'antre. Cela ne devrait pas gêner l'étude des sécrétions du corps de la caillette d'autant plus que l'éventuelle interférence motrice ne devrait affecter que la phase primaire de l'évacuation. De plus, aucun symptôme (gonflement...) indiquant une gène fonctionnelle de la caillette, n'est observé. Afin d'éviter les méfaits de cette vagotomie partielle sur le fonctionnement de l'antre pylorique, nous pourrions envisager de découper la poche abomasale sur la grande courbure de la caillette, en procédant selon la même technique. Toutefois, dans cette région, les terminaisons nerveuses sont intrapariétales, il est donc impossible de les épargner lors de la dissection. La poche abomasale ainsi réalisée ne serait autre qu'une poche dénervée (poche de Heidenhain).

Afin de rester plus près des conditions physiologiques, nous avons conservé intact un plan séro-musculeux de la caillette chez 2 autres veaux. Pour façonner la poche abomasale, la paroi entière est sectionnée sur une face et seulement la muqueuse sur l'autre. La poche n'est délimitée que par la suture de la muqueuse, la caillette 
gardant extérieurement son aspect normal. Cette technique que nous avons appliquée sur la grande courbure, correspond donc à celle de Pavlov, modifiée par Neuwelt, Olson et Necheles (1940) ; elle semble moins traumatisante pour l'animal et permet de conserver l'innervation de l'antre pylorique. Cependant, chez le Veau, elle est moins facile à réaliser que chez le Chien, car la présence de nombreux replis dans le fundus rend difficile l'affrontement des deux plans muqueux. A l'issue de quelques jours, une réperméation s'établit entre la caillette proprement dite ef le petit estomac et il n'est alors plus possible d'obtenir du suc gastrique pur ; il en serait probablement de même si la poche était réalisée sur la petite courbure.

Amour (1930) propose une technique intermédiaire entre les deux précédentes ; appliquée sur la petite courbure, elle conserve intact la séromusculeuse d'une face de l'estomac mais permet d'isoler la poche à la fois par le plan muqueux ef par le plan séromusculeux. Toutefois, la vitalité de certains lambeaux muqueux paraît discutable ef l'auteur ne précise pas la durée d'utilisation expérimentale des poches ainsi obtenues. La technique que nous avons adoptée est plus facile à mettre $\in$ n œuvre. De plus, elle permel de découper très facilement dans le même territoire, une poche totalement dénervée sans modifier profondément la méthode : il suffit pour cela de sectionner les branches du trone vagal dorsal ef du trone vagal ventral.

En conclusion, compte-tenu de l'importance de la durée d'utilisation possible de la poche abomasale, ainsi que de la croissance et de l'état sanitaire satisfaisants des veaux, la technique proposée devrait être un moyen particulièrement intéressant d'étude de la physiologie digestive, sous réserve que le petit estomac et la caillette proprement dite répondent de façon similaire aux stimulus d'ordre physiologique. Elle pourrait aussi être utilisée pour produire des enzymes coagulantes pour la fromagerie à partir de veaux vivants.

Reçu en mai 1977.

Accepté en août 1977.

Remerciements. - A A. du Chalard, Chaire de Zoolechnie, E. N. S. A. de Rennes, pour l'aide apportée dans l'étude anatomique de la caillette.

\section{Références}

AMOUR B. J., 1930. A lesser curvature gastroplasty. Can. med. Assoc. J., 23, 756-761.

ASH R. W., 1961. Acid secretion by the abomasum and its relation to the flow of food material in the sheep. J. Physiol., 156, 93-111.

BERRIDGE N. J., DAVIS J. G., KON P. M., KON S. K., SPRATLING F. R., 1943. The production of rennet from living calves. J. Dairy Res., 13, 145-161.

CISNEROS F., VALLES E., MOCQUOT G., TOMASSONE R., 1972. Obtention de présure à parłir de veaux fistulisés. Le laif, 517, 395-406.

COMLINE R. S., SILVER I. A., STEVEN D. H., 1968. In CODE C. F. Handbook of physiology, section 6. Alimentary canal, vol. 5, 2647-2671, Amer. Physiol. Soc., Washington D.C.

CORRING T., 1975. Adaptation de la sécrétion du pancréas exocrine au régime alimentaire chez le porc, physiologie comparée, étude expérimentale et mécanismes. Thèse doct. d'état ès Sci. Nat., Univ. Paris VI, 100 Pp.

EMAS S., SWAN K. G., JACOBSON E. D., 1967. In CODE C. F., Handbook of physiology, section 6, Alimentary canal, vol. 2, 743-758, Amer. Physiol. Soc., Washington D.C.

GARNOT P., TOULLEC R., THAPON J. L., MARTIN P., MINH-THU-HOANG, MATHIEU C.-M., RIBADEAU-DUMAS B., 1977. Influence of age, dietary protein and weaning on calf abomasal enzymatic secretion. J. Dairy Res., 44, 9-23. 
GROSSKOPF J. F. W., 1954. A simplified operation for the preparation of abomasal pouches in calves. J. Soc. am. Vet. Med. Ass., 25, 59-62.

GROSSKOPF J. F. W., 1959. Some factors affecting the secretion of abomasal juice in young dairy calves. Onderst. J. vet. Res., 28, 133-141.

HABEL R. E., 1975. In GETTY R., The anatomy of the domestic animals, vol. 1, 861-915 et 982-985, Saunders $\mathrm{C}^{\circ}$, Philadelphia.

HILL K. J., GREGORY R. A., 1951. The preparation of gastric pouches in the ruminant. Vet. Rec., 63, 647-652.

IVY A. C., LIM R. K. S., MCCARTY J. E., 1925. Contribution to the physiology of gastric secretion. II. The intestinal phase of gastric secretion. Quart. J. exp. Physiol., 15, 55-64.

KONTUREK S. J., 1974. In JACOBSON E. D., SHANBOURG L. L. Gastrointestinal physiology. Vol. 4, 227-264, Butterworths, London.

MCLEAY L. M., TITCHEN D. A., 1970. Abomasal secretory responses to testing with food and feeding in the sheep. J. Physiol., 206, 605-628.

MIGNON M., BONFILS S., 1972. In MURAT J., Précis de chirurgie expérimentale abdominale et thoracique chez le chien, 41-69, Masson et Cie, Paris.

NAIR P. G., MEHAR SINGH, BHALERAO V. R., 1963. The operation for collecting rennet throught an abomasal fistula. Indian vet. J., 42, 250-254.

NEUWELT F., OLSON W. H., NECHELES H., 1940. A new and simple method for preparing large PAVLOV pouches. Proc. Soc. exp. Biol. Med., 44, 74-80.

ROY J. H. B., STOBO I. J. F., 1975. In MCDONALD J. W., WARNER A. C. I. Digestion and metabolism in the ruminant. Proc. IVth int. Symp. on ruminant physiology, 30-48. Univ. of New England Publ. Unit., Armidale.

RUSEV V., PETROV A., BOCHEVA E., 1971. Peculiarities of rennet secretion in nursing calves in connection with the manner of feeding and milk temperature (en russe). Noutsh. Trud. Zoofech. Fac., 22, 145-156.

SCHUMMER A., WLKENS H., VOLLMERHAUS B., HABERMEHL, K. H., 1976. In NICKEL R., SCHUMMER A., SEIFERLE E., Lehrbuch der Anatomie der Haustiere, Vol. 3, Kreislaufsystem Haut und Hautorgane, 166-183, Verlag Paul Parey, Berlin. 